\title{
A NOTE ON LAGRANGIAN SUBMANIFOLDS OF TWISTOR SPACES AND THEIR RELATION TO SUPERMINIMAL SURFACES
}

\author{
REINIER STORM
}

\begin{abstract}
In this paper a bijective correspondence between superminimal surfaces of an oriented Riemannian 4-manifold and particular Lagrangian submanifolds of the twistor space over the 4-manifold is proven. More explicitly, for every superminimal surface a submanifold of the twistor space is constructed which is Lagrangian for all the natural almost Hermitian structures on the twistor space. The twistor fibration restricted to the constructed Lagrangian gives a circle bundle over the superminimal surface. Conversely, if a submanifold of the twistor space is Lagrangian for all the natural almost Hermitian structures, then the Lagrangian projects to a superminimal surface and is is contained in the Lagrangian constructed from this surface. In particular this produces many Lagrangian submanifolds of the twistor spaces $\mathbb{C} P^{3}$ and $\mathbb{F}_{1,2}\left(\mathbb{C}^{3}\right)$ with respect to both the Kähler structure as well as the nearly Kähler structure. Moreover, it is shown that these Lagrangian submanifolds are minimal submanifolds.
\end{abstract}

\section{INTRODUCTION}

The twistor space of an oriented Riemannian 4-manifold is inspired by Penrose's twistor program and introduced in [2], where the authors use it to classify self-dual solutions of the Yang-Mills equations on $S^{4}$. By now many other spaces which also go by the name of twistor spaces have been constructed; see for example $[22,1,6,3,21]$. These twistor spaces have, among other things, been used to study all kinds of harmonic maps and relate these to holomorphic maps; see [9, 10, 23]. Particular instances of this are superminimal surfaces in 4-manifolds. These are minimal submanifolds and thus yield a harmonic immersion. These types of surfaces had been extensively studied from different points of view; see [4, 11, 16, 17]. Bryant proved in [5] that every compact Riemann surface admits a superminimal immersion into $S^{4}$ using the twistor fibration $\mathbb{C} P^{3} \rightarrow S^{4}$. This inspired many studies of minimal surfaces from this twistor bundle point of view; see for example $[12,8,18,15]$. There are not many results about Lagrangian submanifolds of these twistor spaces. In [7] Lagrangian submanifolds of the Kähler $\mathbb{C} P^{3}$ are constructed, which contain the ones constructed in this paper.

1.1. Results. In [24] all totally geodesic and all homogeneous Lagrangian submanifolds of $\mathbb{F}_{1,2}\left(\mathbb{C}^{3}\right)$, the nearly Kähler manifold of full flags in $\mathbb{C}^{3}$, are classified. The flag manifold $\mathbb{F}_{1,2}\left(\mathbb{C}^{3}\right)$ is the twistor space of $\mathbb{C} P^{2}$. This paper started out as a continuation of the study of Lagrangian submanifolds in $\mathbb{F}_{1,2}\left(\mathbb{C}^{3}\right)$. The relation found

2010 Mathematics Subject Classification. Primary 53C28, Secondary 53C42.

This work is supported by project 3E160361 of the KU Leuven Research Fund and by the EOS research project $3 \mathrm{E} 180171$. 
between superminimal surfaces of $\mathbb{C} P^{2}$ and Lagrangian submanifold of $\mathbb{F}_{1,2}\left(\mathbb{C}^{3}\right)$ turns out to apply for any twistor space $Z$ of an oriented Riemannian 4-manifold. For this we have to define almost Hermitian structures on the twistor space. The twistor spaces come equipped with two natural almost complex structures, which we denote by $J^{ \pm}$; see $(2.2)$ below. In (2.3) we pick a natural family of metrics $g_{\lambda}$ for $\lambda>0$ on $Z$ making $\left(Z, g_{\lambda}, J^{ \pm}\right)$an almost Hermitian space. We will prove the following theorem in Section 4.

Theorem A. For a superminimal surface $\Sigma \subset M^{4}$ there exists a submanifold $L_{\Sigma} \subset Z$ which is a Lagrangian submanifold for all the almost Hermitian structures $\left(g_{\lambda}, J^{ \pm}\right)$simultaneously. This Lagrangian $L_{\Sigma}$ projects under the twistor fibration to $\Sigma$ and the restriction of the twistor fibration to $L_{\Sigma}$ determines a circle bundle over $\Sigma$. Conversely, given a submanifold $L \subset Z$ of the twistor space which is Lagrangian with respect to all the almost Hermitian structures $\left(g_{\lambda}, J^{ \pm}\right)$simultaneously, then the twistor fibration projects $L$ to a surface $\Sigma$ which is superminimal and $L$ is contained in $L_{\Sigma}$.

From the classification of totally geodesic Lagrangian submanifolds of $\mathbb{F}_{1,2}\left(\mathbb{C}^{3}\right)$ in [24] it follows that each of these is congruent under the symmetry group of the nearly Kähler structure of $\mathbb{F}_{1,2}\left(\mathbb{C}^{3}\right)$ to one which projects to a superminimal surface. With the correspondence of Theorem A we obtain infinitely many new examples of Lagrangian submanifolds of both the Kähler and nearly Kähler structures on $\mathbb{F}_{1,2}\left(\mathbb{C}^{3}\right)$ and $\mathbb{C} P^{3}$ by relating them to superminimal surfaces. Finally, it is shown that the submanifolds $L_{\Sigma} \subset\left(Z, g_{\lambda}\right)$ are minimal submanifolds.

\section{TWISTOR SPACE}

In this section we give a short introduction to the twistor fibration over a 4manifold. Let $\left(M^{4}, g\right)$ be an oriented Riemannian 4-manifold. For a given complex structure $J \in \operatorname{End}\left(T_{x} M\right)$ which is compatible with the metric, i.e. $J \in O\left(T_{x} M\right)$, let $\omega_{J}(-,-)=g(J-,-) \in \Lambda^{2} T_{x}^{*} M$. The complex structure $J$ is said to be compatible with the orientation if $\omega_{J} \wedge \omega_{J}$ is equal to the orientation of $M^{4}$ at $x$, which is denoted as $\omega_{J} \wedge \omega_{J} \gg 0$.

Definition 2.1. The twistor bundle $\pi: Z \rightarrow M^{4}$ of an oriented Riemannian manifold $\left(M^{4}, g\right)$ is the bundle whose fiber over a point $x \in M^{4}$ consists of all complex structures on the vector space $T_{x} M^{4}$ which are compatible with the Riemannian metric and the orientation, i.e.

$$
\pi^{-1}(x)=\left\{J \in \operatorname{End}\left(T_{x} M^{4}\right): J^{2}=-1, J^{*} g=g \text { and } \omega_{J} \wedge \omega_{J} \gg 0\right\} .
$$

The fiber is isomorphic to $S O(4) / U(2) \cong \mathbb{C} P^{1}$.

Alternatively, the twistor bundle can be defined as the associated bundle of the principal $S O(4)$-frame bundle $\mathcal{F}$ of $\left(M^{4}, g\right)$ by

$$
Z \cong \mathcal{F} \times_{S O(4)} S O(4) / U(2)
$$

Here $S O(4) / U(2)$ is identified with all complex structures $J$ on $\mathbb{R}^{4}$ such that $J \in$ $S O(4)$ and $\omega_{J} \wedge \omega_{J} \gg 0$. Elements of $Z$ are equivalence classes $[u, J]$, where $u: \mathbb{R}^{4} \rightarrow T_{x} M$ is a frame and $J$ is a complex structure on $\mathbb{R}^{4}$. The equivalence relation is given by $[u \cdot g, J] \sim[u, g \cdot J]$ for $g \in S O(4)$. The Levi-Civita connection 
on $M^{4}$ induces a horizontal subbundle $T^{h} Z$ which is complementary to the vertical subbundle $T^{v} Z$ of the tangent space of $Z$, i.e.

$$
T Z=T^{v} Z \oplus T^{h} Z .
$$

The derivative of $\pi$ restricted to $T^{h} Z$ yields an isomorphism between $T_{I}^{h} Z$ and $T_{\pi(I)} M$ for all $I \in Z$. Two natural almost complex structures on $Z$ are defined by

$$
J_{I}^{ \pm}= \pm J_{\mathbb{C} P^{1}}+I \in \operatorname{End}\left(T_{I} Z\right)
$$

where $J_{\mathbb{C} P^{1}}$ is the natural complex structure on the fiber $\mathbb{C} P^{1}$ and $I \in Z$ is a complex structure on the vector space $T_{\pi(I)} M^{4} \cong T_{I}^{h} Z$. In [2] it is shown that $J^{+}$is integrable if and only if $\left(M^{4}, g\right)$ is anti-self-dual, which is an essential ingredient for the classification of instantons of $S^{4}$ in [2]. Moreover, $J^{+}$is conformally invariant; see [2]. The almost complex structure $J^{-}$is never integrable; see [23].

A third point of view on the twistor space is the taken in [1]. Where the twistor space is naturally identified as a quotient of $\mathcal{F}$, namely $Z:=\mathcal{F} / U(2)$, where $U(2) \subset$ $S O(4)$ is the stabilizer subgroup of some fixed complex structure $J_{0}$ on $\mathbb{R}^{4}$. The natural isomorphism $\mathcal{F} / U(2)$ to our previous definition of the twistor space is given by

$$
u \cdot U(2) \mapsto\left[u, J_{0}\right]
$$

This map does not depend on the representative $u$ of the coset and yields a bundle isomorphism. Let $\mathfrak{s o}(5)=\mathfrak{s o}(4) \oplus \mathfrak{p}$ be the Cartan decomposition. Let $\mathfrak{u}(2) \subset \mathfrak{s o}(4)$ be the stabilizer algebra of $J_{0}$. This yields a decomposition

$$
\mathfrak{s o}(5)=\mathfrak{u}(2) \oplus \mathfrak{n} \oplus \mathfrak{p},
$$

where $\mathfrak{n} \subset \mathfrak{s o}(4)$ is the orthogonal complement of $\mathfrak{u}(2)$ with respect to the Killing form. Throughout this work $B$ will denote the Killing form on $\mathfrak{s o}(5)$. We will denote $\mathfrak{m}:=\mathfrak{n} \oplus \mathfrak{p}$ and $\mathfrak{h}:=\mathfrak{u}(2) \subset \mathfrak{s o}(5)$. The advantage of this point of view is that $Z$ comes equipped with a principal $U(2)$-bundle $\mathcal{F} \rightarrow Z$ together with a Cartan connection

$$
\phi: \mathcal{F} \rightarrow \mathfrak{s o}(5)=\mathfrak{h} \oplus \mathfrak{m},
$$

which is just the Levi-Civita connection on the principal frame bundle combined with the soldering form. The homogeneous space $S O(5) / U(2)$ is an adjoint orbit of a central element $z \in \mathcal{Z}(U(2))$. This induces a Kähler-Einstein structure on $S O(5) / U(2)$. The metric at the identity coset is given by

$$
g_{K}=-\left.2 B\right|_{\mathfrak{n}} \oplus-\left.B\right|_{\mathfrak{p}} .
$$

The corresponding symplectic form $\omega_{K}$ is the Kirillov-Kostant-Souriau symplectic form and is up to a scaling factor given by $\operatorname{ad}(z)$. The associated complex structure is above denoted by $J^{+}$. The almost complex structure $J^{-}$is in this setting given by

$$
\left.J^{-}\right|_{\mathfrak{p}}=\left.J^{+}\right|_{\mathfrak{p}} \quad \text { and }\left.\quad J^{-}\right|_{\mathfrak{n}}=-\left.J^{+}\right|_{\mathfrak{n}} .
$$

More generally, The model space $S O(5) / U(2)$ comes equipped with a natural family of $U(3)$ structures

$$
\left(g_{\lambda}, J^{ \pm}\right)
$$

where $g_{\lambda}=-\left.\frac{1}{\lambda^{2}} B\right|_{\mathfrak{n}} \oplus-\left.B\right|_{\mathfrak{p}}$. The nearly Kähler structure on $S O(5) / U(2)$ is given by $\left(S O(5) / U(2), g_{1}, J^{-}\right)$. These $U(3)$-structures can be pulled back to $Z$ by the Cartan connection. Moreover, these structures are parallel with respect to this 
connection. In the same way it is possible to induce a natural family of $S U(3)$ structures on $Z$. Let $\Upsilon$ denote the natural complex volume form induces from the nearly Kähler structure on $S O(5) / U(2)$. Then

$$
\left(g_{\lambda}, J^{ \pm}, \lambda \Upsilon\right)
$$

defines a family of $S U(3)$-structures on $Z$. The results here do not depend on the particular value of $\lambda$. Some obvious properties of $g_{\lambda}$ are that $\left(Z, g_{\lambda}, J^{ \pm}\right)$is an almost Hermitian manifold and the fibers $\pi^{-1}(x) \subset Z$ are totally geodesic submanifolds.

\section{SUPERMINIMAL SURFACES}

Let $\Sigma$ be an oriented 2-dimensional surface in a 4-dimensional oriented Riemannian manifold $\left(M^{4}, g\right)$. Let $N \Sigma \subset T M^{4}$ be the normal bundle of $T \Sigma$. Let $J_{0}$ be the complex structure on $\left.T M^{4}\right|_{\Sigma}$ defined by a rotation by $\frac{\pi}{2}$ in $T_{x} \Sigma$ and a rotation by $\frac{\pi}{2}$ in $N_{x} \Sigma$. This determines a lift $F_{0}: \Sigma \rightarrow Z$ of the inclusion $i: \Sigma \rightarrow M^{4}$ :

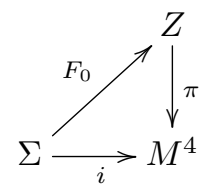

with $\pi \circ F_{0}=i$.

Remark 3.1. For an oriented surface $\Sigma \subset M^{4}$ there is a natural $U(2)$-reduction of the restricted frame bundle $\left.\mathcal{F}\right|_{\Sigma}$. Whenever we mention the group $U(2)$ below we are referring to this $U(2)$-reduction given by the complex structure $F_{0}$ along $\Sigma$.

Fix an oriented orthonormal local frame $u=\left(e_{1}, e_{2}, e_{3}, e_{4}\right)$ of $T M^{4}$ such that $\left(e_{1}, e_{2}\right)$ is an oriented basis of $T \Sigma$. The complex structure $J_{0}$ is determined by $J_{0}\left(e_{1}\right)=e_{2}$ and $J_{0}\left(e_{3}\right)=e_{4}$. In the identification of $Z$ with the associated bundle as in (2.1) the lift $F_{0}: \Sigma \rightarrow Z$ is locally given by

$$
F_{0}(x)=\left[u(x), J_{0}\right],
$$

where we make some slight abuse of notation by denoting the complex structure on $\mathbb{R}^{4}$ corresponding to $J_{0}$ in the frame $u$ also by $J_{0}$.

Definition 3.2. An oriented surface $\Sigma \subset M^{4}$ is superminimal if the vertical component $\left(\mathrm{d} F_{0}\right)^{v}$ of $\mathrm{d} F_{0}$ vanishes.

Remark 3.3. It is easy to see that a superminimal surface is in particular minimal. An oriented minimal surface $\Sigma \subset M^{4}$ is superminimal if in addition $F_{0}: \Sigma \rightarrow$ $\left(Z, J^{+}\right)$is holomorphic with respect to the complex structure of $\Sigma$ induced from the conformal structure; see [12].

The following lemma is quite trivial, but nevertheless it is a useful equivalent condition for superminimal surfaces.

Lemma 3.4. For an oriented surface $\Sigma \subset M^{4}$ the following are equivalent

(i) the surface $\Sigma$ is superminimal,

(ii) the complex structure $F_{0}$ on $\left.T M\right|_{\Sigma}$ is parallel, i.e. $\operatorname{Hol}\left(\left.\nabla\right|_{\Sigma}\right) \subset U(2)$, where $U(2)$ is defined with respect to $F_{0}$. 
Proof. Let $\gamma: I \rightarrow \Sigma$ be a curve. We will use the description of $Z$ as associated bundle $\mathcal{F} \times{ }_{S O(4)} S O(4) / U(2)$. Thus the local formula (3.1) gives

$$
\begin{aligned}
\left(\mathrm{d}_{\gamma^{\prime}(t)} F_{0}\right)^{v} & =\left(\frac{d}{d t}\left[u(\gamma(t)), J_{0}\right]\right)^{v}=\left(\frac{d}{d t}\left[u^{h}(\gamma(t)) \cdot g(t), J_{0}\right]\right)^{v} \\
& =\left(\frac{d}{d t}\left[u^{h}(\gamma(t)), g(t) J_{0}\right]\right)^{v}=\frac{d}{d t} g(t) J_{0},
\end{aligned}
$$

where $u^{h}(\gamma(t))$ is a horizontal lift of $\gamma$ and $g(t) \in S O(4)$ is the parallel translation along $\gamma$ with respect to $\left.\nabla\right|_{\Sigma}$ in the frame $u$. In the last equality we identified the fiber $\pi^{-1}(\gamma(t))$ with the space of complex structures on $\mathbb{R}^{4}$ via $u^{h}(\gamma(t))$. This implies that $g(t) \in \operatorname{stab}\left(J_{0}\right) \cong U(2) \subset S O(4)$ if and only if $\left(\mathrm{d}_{\gamma^{\prime}(t)} F_{0}\right)^{v}=0$. Consequently, the holonomy of the $\left.\nabla\right|_{\Sigma}$ is contained in $U(2)$ if and only if $\Sigma$ is superminimal.

There are a couple of interesting other equivalent formulations of superminimal surfaces. For example a surface $\Sigma \subset M^{4}$ is superminimal if and only if the indicatrix, also known as the curvature ellipse, of $\Sigma$ is a circle centered at zero. Or alternatively, $\Sigma$ is superminimal if and only if it is negatively oriented-isoclinic. This was proven in [17] for $M=\mathbb{R}^{4}$ and the general case is proven in [12]. These two properties of surfaces in a 4-manifold have been studied in the beginning of the 20th century. The equivalence of these other characterizations to Definition 3.2 is proven in [12].

\section{A CORRESPONDENCE BETWEEN LAGRANGIANS AND SUPERMINIMAL SURFACES}

Let $\Sigma \subset M^{4}$ be a superminimal surface. Below we will construct a submanifold $L_{\Sigma} \subset Z$, which is Lagrangian with respect to all of the almost Hermitian structures $\left(Z, g_{\lambda}, J^{ \pm}\right)$. The restriction of the twistor fibration $\left.\pi\right|_{L_{\Sigma}}: L_{\Sigma} \rightarrow \Sigma$ will be a circle bundle and the fibers of $\left.\pi\right|_{L_{\Sigma}}$ are geodesics in $\left(Z, g_{\lambda}\right)$. In particular $L_{\Sigma}$ is a $\lambda$-ruled Lagrangian submanifold in the sense of [19].

Let $\Sigma$ be a superminimal surface in $\left(M^{4}, g\right)$ and let $N \Sigma \subset T M^{4}$ denote the normal bundle of $T \Sigma$. Let $u=\left(e_{1}, e_{2}, e_{3}, e_{4}\right)$ be an oriented orthonormal local frame of $M$ such that $\left(e_{1}, e_{2}\right)$ is an oriented orthonormal basis of $T \Sigma$. Define a submanifold $L_{\Sigma}$ of $Z$ as a bundle over $\Sigma$ by

$$
L_{\Sigma} \cap \pi^{-1}(x):=\left\{J \in Z_{x}\left(M^{4}\right): J\left(T_{x} \Sigma\right)=N_{x} \Sigma\right\} .
$$

Note that $\left.\pi\right|_{L_{\Sigma}}: L_{\Sigma} \rightarrow \Sigma$ is a circle bundle and the fibers can in terms of the frame be expressed as the complex structures determined by

$$
J_{\theta}\left(e_{1}\right)=\cos (\theta) e_{3}+\sin (\theta) e_{4}, \quad J_{\theta}\left(e_{2}\right)=\sin (\theta) e_{3}-\cos (\theta) e_{4},
$$

for $\theta \in S^{1}$. Just as we did with $J_{0}$ we will also denote the complex structure on $\mathbb{R}^{4}$ corresponding to $J_{\theta}$ in the frame $u$ by $J_{\theta}$. These complex structures at a tangent space $T_{x} M^{4}$ for $x \in \Sigma$ can be depicted as in Figure 1. The complex structures $J_{\theta}$ form the equator of the fiber $\pi^{-1}(x)$ with respect to the poles $\left\{J_{0},-J_{0}\right\}$.

Remark 4.1. The important property of the equator $S_{x}^{1}=\left\{J_{\theta}\right\}_{\theta \in S^{1}}$ of complex structures is that the subalgebra which preserves this equator is exactly the stabilizer algebra of $J_{0}$, i.e. $\mathfrak{u}(2) \subset \mathfrak{s o}(4)$.

Proof of Theorem A. Suppose $\Sigma$ is a superminimal surface. Fix an oriented orthonormal local frame $u=\left(e_{1}, e_{2}, e_{3}, e_{4}\right)$ of $T M^{4}$ such that $\left(e_{1}, e_{2}\right)$ is an oriented basis of $T \Sigma$. First we show that the tangent space of $L_{\Sigma}$ is compatible 


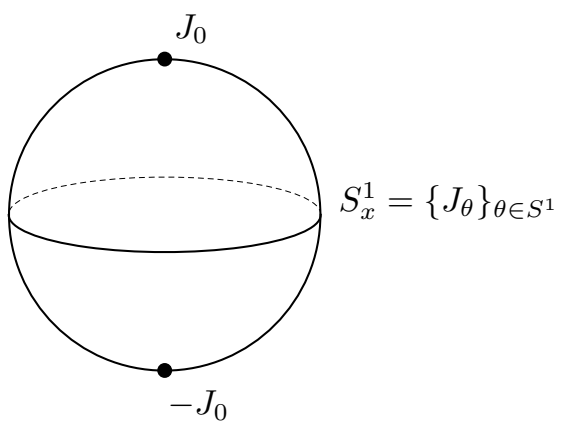

Figure 1. Complex structures of $T_{x} M^{4}$ at a point $x \in \Sigma$.

with the splitting of $Z$ into the vertical and horizontal subbundles. A point of $L_{\Sigma}$ is in the frame $u$ expressed as $J_{\theta}$ for some $\theta$. Note that tangent space of $L_{\Sigma}$ which is contained in the vertical subbundle of $T Z$ is equal to $T_{J_{\theta}} S_{x}^{1}$, where $\pi\left(J_{\theta}\right)=x \in \Sigma$. Let $F_{\theta}: \Sigma \rightarrow L_{\Sigma} \subset Z$ be the local lift given by $F_{\theta}(y)=\left[u(y), J_{\theta}\right]$. By Lemma 3.4 we know that parallel translation in $Z$ along a curve in $\Sigma$ preserves $J_{0}$. Thus by Remark 4.1 it also preserves the subbundle $L_{\Sigma} \subset Z$. From this we obtain that the vertical component of $\mathrm{d} F_{\theta}(x)$ is contained in $T_{J_{\theta}} S_{x}^{1}$. Clearly $\operatorname{im}\left(\mathrm{d} F_{\theta}(x)\right) \oplus T_{J_{\theta}} S_{x}^{1}=T_{J_{\theta}} L_{\Sigma}$ holds. Consequently, the tangent space of $L_{\Sigma}$ at $J_{\theta}$ splits into a vertical and a horizontal subspace, i.e.

$$
T_{J_{\theta}} L_{\Sigma}=T_{J_{\theta}}^{v} L_{\Sigma} \oplus T_{J_{\theta}}^{h} L_{\Sigma}
$$

Let $X \in T_{J_{\theta}} L_{\Sigma}$ and write it as $X=X^{v}+X^{h}$, with $X^{v} \in T_{J_{\theta}}^{v} L_{\Sigma}$ and $X^{h} \in T_{J_{\theta}}^{h} L_{\Sigma}$ and similarly for $Y \in T_{J_{\theta}} L_{\Sigma}$. We have

$$
g_{\lambda}\left(J^{ \pm}(X), Y\right)= \pm g_{\lambda}\left(J_{\mathbb{C} P^{1}}\left(X^{v}\right), Y^{v}\right)+g_{\lambda}\left(J_{\theta}\left(X^{h}\right), Y^{h}\right)=0,
$$

where $J_{\mathbb{C} P^{1}}\left(X^{v}\right)$ is perpendicular to $Y^{v}$ because $X^{v}$ and $Y^{v}$ are linearly dependent and $J_{\theta}\left(X^{h}\right)$ is perpendicular to $Y^{h}$ because $J_{\theta}$ maps $T \Sigma$ to $N \Sigma$. Thus $J^{ \pm}\left(T L_{\Sigma}\right)$ is perpendicular to $T L_{\Sigma}$ with respect to the metric $g_{\lambda}$. We conclude that $L_{\Sigma} \subset$ $\left(Z, g_{\lambda}, J^{ \pm}\right)$is a Lagrangian submanifold with respect to all the almost Hermitian structures.

Conversely, suppose we are given a submanifold $L \subset\left(Z, g_{\lambda}\right)$ which is Lagrangian for both $J^{+}$and $J^{-}$. Let $\omega_{ \pm}$be the Kähler forms of these almost Hermitian structures, i.e.

$$
\omega_{ \pm}(X, Y)=g_{\lambda}\left(J^{ \pm}(X), Y\right)
$$

Let $\omega_{ \pm}=\omega_{ \pm}^{v}+\omega_{ \pm}^{h}$ be the decomposition into their vertical and horizontal parts. The vertical and horizontal parts of $\omega_{ \pm}$satisfy $\omega_{+}^{v}=-\omega_{-}^{v}$ and $\omega_{+}^{h}=\omega_{-}^{h}$. Thus both 2forms $\omega_{+}^{v}$ and $\omega_{+}^{h}$ vanish on $L$. The projection of $T_{I} L$ on the horizontal distribution $T_{I}^{h} Z$ is a Lagrangian subspace for every $I \in L$ with respect to the restricted almost Hermitian structure, because $\omega_{+}^{h}$ vanish on $L$. Thus this projection necessarily has a non-trivial kernel. The projection of $T_{I} L$ onto the vertical distribution $T_{I}^{v} Z$ can be at most 1-dimensional, because $\omega_{+}^{v}$ vanishes on $L$. Consequently, the projection of $T L$ onto the vertical distribution is equal to its intersection with the vertical distribution and the splitting

$$
T_{I} L=T_{I}^{v} L \oplus T_{I}^{h} L
$$


holds. In particular $\Sigma:=\pi(L) \subset M^{4}$ is a 2-dimensional surface. As before we fix some oriented orthonormal local frame $u=\left(e_{1}, e_{2}, e_{3}, e_{4}\right)$ on $M^{4}$ such that $\left(e_{1}, e_{2}\right)$ is an oriented basis of $T \Sigma$. A complex structure in $L$ is then of the form $J_{\theta}$ as in (4.1) for some $\theta$, because $L$ is Lagrangian. A lift of $\left.\pi\right|_{L}: L \rightarrow \Sigma$ is given by $F_{\theta}=\left[u, J_{\theta}\right]: \Sigma \rightarrow L$. This implies $\left(\mathrm{d} F_{\theta}\right)^{v} \in T_{J_{\theta}}^{v} L=T_{J_{\theta}} S_{x}^{1}$ and by Remark 4.1 this implies that the holonomy group of $\left.\nabla\right|_{\Sigma}$ is contained in $U(2)$. Therefore, by Lemma 3.4 we obtain that $\Sigma$ is superminimal.

4.1. Minimal Submanifolds. For Lagrangian submanifolds in nearly Kähler manifolds it is known that they are minimal submanifolds; see [13]. Apart from these two homogeneous nearly Kähler twistor spaces the almost Hermitian manifolds $\left(Z, g_{\lambda}, J^{ \pm}\right)$are never nearly Kähler. However, in this section we will show that the Lagrangians constructed in Section 4 are minimal submanifolds for all of these spaces $\left(Z, g_{\lambda}\right)$ simultaneously.

In this section we denote the Levi-Civita connection on $Z$ by $\nabla^{g}$, where the index $\lambda$ is excluded from the notation. The connection on $Z$ obtained from the Cartan connection $\phi: T \mathcal{F} \rightarrow \mathfrak{s o}(5)=\mathfrak{h} \oplus \mathfrak{m}$ on $\mathcal{F} \rightarrow Z$ is denoted by $\nabla$; see also Section 2 and [1] for more details. Furthermore, let $A=\nabla^{g}-\nabla$. We will use $\nabla$ to compute the mean curvature. Let

$$
B_{0}:=\frac{1}{\sqrt{2}}\left(\begin{array}{cccc}
1 & 0 & 0 & -1 \\
0 & 1 & -1 & 0 \\
0 & 1 & 1 & 0 \\
1 & 0 & 0 & 1
\end{array}\right)
$$

and $B_{\theta}:=\exp \left(\theta J_{0}\right) B_{0}$ for $\theta \in S^{1}$. These matrices are chosen such that $B_{\theta} J_{0} B_{\theta}^{-1}=$ $J_{\theta}$. Let $u$ be an adapted frame along $\Sigma$ as before. We define a lift $\hat{u}: L_{\Sigma} \rightarrow \mathcal{F}$ of $\pi: \mathcal{F} \rightarrow Z$ by

This is a local lift, because

$$
\hat{u}\left(\left[u(x), J_{\theta}\right]\right)=u(x) \cdot B_{\theta} .
$$

$$
\pi\left(\hat{u}\left(\left[u(x), J_{\theta}\right]\right)\right)=\left[u(x) \cdot B_{\theta}, J_{0}\right]=\left[u(x), B_{\theta} \cdot J_{0}\right]=\left[u(x), J_{\theta}\right] .
$$

Let us denote $p_{x, \theta}:=\hat{u}\left(\left[u(x), J_{\theta}\right]\right)$. Let $R_{h}: \mathcal{F} \rightarrow \mathcal{F}$ denote the right principal bundle action by an element $h \in S O(4)$. We define an orthonormal frame of $L_{\Sigma}$ as follows. Let

$$
\hat{v}_{3}\left(p_{x, \theta}\right)=\frac{\lambda}{\sqrt{12}} \frac{d}{d \theta} R_{B_{\theta}}(u(x)) \in T_{p_{x, \theta}} \mathcal{F}
$$

and for $i=1,2$ we let

$$
\hat{v}_{i}\left(p_{x, \theta}\right)=R_{B_{\theta *}} u_{*} e_{i} \in T_{p_{x, \theta}} \mathcal{F} \text {. }
$$

Furthermore, if we put $v_{i}=\pi_{*} \hat{v}_{i}$, then $\left(v_{1}, v_{2}, v_{3}\right)$ is an orthonormal frame for $L_{\Sigma}$ with respect to the metric $g_{\lambda}$. The vector $v_{3}$ is vertical for the bundle $Z \rightarrow M$ and the vectors $v_{1}$ and $v_{2}$ are horizontal.

The mean curvature will by computed by

$$
\sum_{i=1}^{3}\left(\nabla_{v_{i}} v_{i}\right)^{\perp}=\sum_{i=1}^{3}\left(\nabla_{v_{i}}^{g} v_{i}\right)^{\perp}+\left(A\left(v_{i}\right) v_{i}\right)^{\perp} .
$$

We will show that $\sum_{i=1}^{3}\left(\nabla_{v_{i}} v_{i}\right)^{\perp}=\sum_{i=1}^{3}\left(A\left(v_{i}\right) v_{i}\right)^{\perp}=0$ and thus that the mean curvature vector of $L_{\Sigma} \subset\left(Z, g_{\lambda}\right)$ vanishes. 
First we consider $\sum_{i=1}^{3}\left(\nabla_{v_{i}} v_{i}\right)^{\perp}$. We identify a vector field $X$ on $Z$ with its $U(2)$-equivariant function $\Psi(X): \mathcal{F} \rightarrow \mathfrak{m}$, where $\mathfrak{m}$ is defined in Section 2. The covariant derivative $\nabla$ can be expressed in terms of the Cartan connection by

$$
\Psi\left(\nabla_{v_{i}} v_{i}\right)=\mathrm{d}_{\hat{v}_{i}} \phi_{\mathfrak{m}}\left(\hat{v}_{i}\right)+\phi_{\mathfrak{h}}\left(\hat{v}_{i}\right) \cdot \phi_{\mathfrak{m}}\left(\hat{v}_{i}\right),
$$

where $\cdot$ denotes the restricted adjoint action of $\mathfrak{h}$ on $\mathfrak{m}$. First of all we have $\phi\left(\hat{v}_{3}\right)=$ $\frac{\lambda}{\sqrt{12}} \operatorname{Ad}\left(B_{0}\right)^{-1} \cdot J_{0} \in \mathfrak{n}$, where $J_{0}$ is seen as an element of $\mathfrak{h} \subset \mathfrak{s o}(5)$. This is a constant function and $\phi_{\mathfrak{h}}\left(\hat{v}_{3}\right)=0$, thus we find $\nabla_{v_{3}} v_{3}=0$. Furthermore,

$$
\begin{aligned}
\sum_{i=1}^{2} \Psi\left(\nabla_{v_{i}} v_{i}\right)^{\perp} & =\sum_{i=1}^{2}\left(\mathrm{~d}_{\hat{v}_{i}} \phi_{\mathfrak{m}}\left(R_{B_{\theta *}} u_{*} e_{i}\right)+\phi_{\mathfrak{h}}\left(R_{B_{\theta *}} u_{*} e_{i}\right) \cdot \phi_{\mathfrak{m}}\left(R_{B_{\theta *}} u_{*} e_{i}\right)\right)^{\perp} \\
& =\sum_{i=1}^{2}\left(\operatorname{Ad}\left(B_{\theta}\right)^{-1} \mathrm{~d}_{\hat{v}_{i}} \phi_{\mathfrak{m}}\left(u_{*} e_{i}\right)+\operatorname{Ad}\left(B_{\theta}\right)^{-1} \phi_{\mathfrak{h}}\left(u_{*} e_{i}\right) \operatorname{Ad}\left(B_{\theta}\right) \cdot \phi_{\mathfrak{m}}\left(R_{B_{\theta *}} u_{*} e_{i}\right)\right)^{\perp} \\
& =\left(\operatorname{Ad}\left(B_{\theta}\right)^{-1} \sum_{i=1}^{2} \mathrm{~d}_{\hat{v}_{i}} \phi_{\mathfrak{m}}\left(u_{*} e_{i}\right)+\phi_{\mathfrak{h}}\left(u_{*} e_{i}\right) \cdot \phi_{\mathfrak{m}}\left(u_{*} e_{i}\right)\right)^{\perp} \\
& =0,
\end{aligned}
$$

where $B_{\theta} \in O(4)$ and in the last equality the sum expresses the mean curvature vector of $\Sigma \subset M$ which is zero, because $\Sigma$ is superminimal.

Next we consider $\sum_{i=1}^{3} A\left(v_{i}\right) v_{i}$. The connection form $A$ with respect to the Levi-Civita connection can be expressed in terms of the torsion $T$ of $\nabla$ as

$$
g_{\lambda}(A(X) Y, Z)=\frac{1}{2}(T(X, Y, Z)-T(Y, Z, X)+T(Z, X, Y)) .
$$

Thus we find

$$
\begin{aligned}
\sum_{i=1}^{3} g_{\lambda}\left(A\left(v_{i}\right) v_{i}, Z\right) & =\sum_{i=1}^{3} \frac{1}{2}\left(T\left(v_{i}, v_{i}, Z\right)-T\left(v_{i}, Z, v_{i}\right)+T\left(Z, v_{i}, v_{i}\right)\right) \\
& =\sum_{i=1}^{3} T\left(Z, v_{i}, v_{i}\right) .
\end{aligned}
$$

The torsion tensor $T$ is identified with an equivariant function $\hat{T}: \mathcal{F} \rightarrow \Lambda^{2} \mathfrak{m}^{*} \otimes \mathfrak{m}$, which is given by

$$
\hat{T}=\kappa_{\mathfrak{m}}-t,
$$

where $\kappa_{\mathfrak{m}}$ is the $\mathfrak{m}$-component of the curvature function of $\phi$ and $t$ is the constant function which takes the value $g_{\lambda}(t(x, y), z)=g_{\lambda}([x, y], z)$. The torsion of the Cartan connection is given by

$$
\kappa_{\mathfrak{m}}=\kappa_{\mathfrak{n}}+\kappa_{\mathfrak{p}}=R_{\mathfrak{n}}-\left[\phi_{\mathfrak{n}}, \phi_{\mathfrak{p}}\right],
$$

where $\kappa_{\mathfrak{n}}=R_{\mathfrak{n}}$ is the Riemannian curvature tensor of $\left(M^{4}, g\right)$ followed by a projection onto $\mathfrak{n}$; see also [1].

Lemma 4.2. If either $X \in \mathfrak{n}$ or $X \in \mathfrak{p}$, then for all $Z \in \mathfrak{m}$ the following hold:

(i) $g_{\lambda}(t(Z, X), X)=0$,

(ii) $g_{\lambda}\left(R(Z, X)_{\mathfrak{n}}, X\right)=0$,

(iii) $g_{\lambda}\left(\left[\phi_{n}(Z), \phi_{\mathfrak{p}}(X)\right]-\left[\phi_{\mathfrak{n}}(X), \phi_{\mathfrak{p}}(Z)\right], X\right)=0$. 
Proof. For (i) we define $t_{\lambda} \in \bigotimes^{3} \mathfrak{m}^{*}$ by $t_{\lambda}(X, Y, Z)=g_{\lambda}([X, Y], Z)$. Note that $t_{1} \in$ $\Lambda^{3} \mathfrak{m}^{*}$, because $g_{1}$ is equal to minus the Killing form of $\mathfrak{s o}(5)$ and thus invariant under the adjoint action. Suppose $Z \in \mathfrak{n}$ and $X \in \mathfrak{n}$, then $t_{\lambda}(Z, X, X)=\lambda t_{1}(Z, X, X)=0$. If $Z \in \mathfrak{p}$ and $X \in \mathfrak{n}$, then $[Z, X]_{\mathfrak{m}} \in \mathfrak{p}$ and thus $t_{\lambda}(Z, X, X)=0$. Similarly, for $Z \in \mathfrak{p}$ and $X \in \mathfrak{p}$ we have $[Z, X]_{\mathfrak{m}} \in \mathfrak{n}$ and thus $t_{\lambda}(Z, X, X)=0$. Lastly, if $Z \in \mathfrak{n}$ and $X \in \mathfrak{p}$, then $t_{\lambda}(Z, X, X)=t_{1}(Z, X, X)=0$. By a similar argument we obtain (iii) holds.

The Riemannian curvature tensor is a horizontal tensor on $\mathcal{F}$, thus $R(X, Y)=0$ if either $X \in \mathfrak{n}$ or $Y \in \mathfrak{n}$. From this (ii) follows.

From this lemma we obtain

$$
\sum_{i=1}^{3} T\left(Z, v_{i}, v_{i}\right)=0,
$$

for all $Z$. We conclude that the Lagrangian submanifold $L_{\Sigma} \subset\left(Z, g_{\lambda}\right)$ is a minimal submanifold.

Remark 4.3. A $k$-form $\alpha$ which satisfies $\alpha(\eta) \leq 1$ for every normalized $k$-multivector is called a generalized calibration; see [13]. A $k$-dimensional submanifold $L$ which satisfies $\alpha\left(\eta_{x}\right)=1$ for every $x \in L$ is called calibrated, where $\eta_{x}$ is a normalized top degree multivector on $T_{x} L$. If in addition the form $\alpha$ satisfies $\mathrm{d} \alpha=0$, then it is called a calibration form. Calibrated submanifolds of calibrations are automatically volume minimizing within their homology class; see [14]. An interesting phenomenon occurs for calibrated submanifolds of nearly Kähler manifolds. In [13] it is shown that special Lagrangian submanifolds are automatically minimal and in [20] it is shown that pseudo-holomorphic curves are also minimal. Furthermore, there are no pseudo-holomorphic surfaces of (strict) nearly Kähler structures. Thus every calibrated submanifold of a natural generalized calibration form of a nearly Kähler manifold is minimal. We have shown that the Lagrangian submanifolds $L_{\Sigma}$, which we constructed here are also minimal. It might be interesting to point out that they are also calibrated by the complex volume form $\lambda \Upsilon$, which was defined in (2.4) and is a generalized calibration.

\section{REFERENCES}

[1] D. V. Alekseevsky and M. M. Graev. G-structures of twistor type and their twistor spaces. J. Geom. Phys., 10(3):203-229, 1993. doi:10.1016/0393-0440(93)90015-7.

[2] M. F. Atiyah, N. J. Hitchin, and I. M. Singer. Self-duality in four-dimensional Riemannian geometry. Proc. Roy. Soc. London Ser. A, 362(1711):425-461, 1978. doi:10.1098/rspa. 1978. 0143.

[3] L. Bérard-Bergery and T. Ochiai. On some generalizations of the construction of twistor spaces. In Global Riemannian geometry (Durham, 1983), Ellis Horwood Ser. Math. Appl., pages 52-59. Horwood, Chichester, 1984.

[4] O. Borůvka. Sur une classe de surfaces minima plongées dans un espace à quatre dimensions à courbure constante. Bulletin int. Acad. Tchèque Sci., 29:256-277, 1928.

[5] R. L. Bryant. Conformal and minimal immersions of compact surfaces into the 4sphere. J. Differential Geom., 17(3):455-473, 1982. URL: http://projecteuclid.org/euclid.jdg/ 1214437137.

[6] F. E. Burstall and J. H. Rawnsley. Twistor theory for Riemannian symmetric spaces, volume 1424 of Lecture Notes in Mathematics. Springer-Verlag, Berlin, 1990. With applications to harmonic maps of Riemann surfaces. doi:10.1007/BFb0095561.

[7] I. Castro and L. Vrancken. Minimal Lagrangian submanifolds in $\mathbb{C} P^{3}$ and the sinh-Gordon equation. Results Math., 40(1):130-143, Oct 2001. doi:10.1007/BF03322703. 
[8] Q.-S. Chi and X. Mo. The moduli space of branched superminimal surfaces of a fixed degree, genus and conformal structure in the four-sphere. Osaka J. Math., 33(3):669-696, 1996. URL: http://projecteuclid.org/euclid.ojm/1200787096.

[9] J. Eells and S. Salamon. Constructions twistorielles des applications harmoniques. C. R. Acad. Sci. Paris Sér. I Math., 296(15):685-687, 1983. doi:10.1142/9789814360197_0015.

[10] J. Eells and S. Salamon. Twistorial construction of harmonic maps of surfaces into fourmanifolds. Ann. Scuola Norm. Sup. Pisa Cl. Sci. (4), 12(4):589-640 (1986), 1985. URL: http://www.numdam.org/item?id=ASNSP_1985_4_12_4_589_0.

[11] L. P. Eisenhart. Minimal Surfaces in Euclidean Four-Space. Amer. J. Math., 34(3):215-236, 1912. doi:10.2307/2370220.

[12] T. Friedrich. On surfaces in four-spaces. Ann. Global Anal. Geom., 2(3):257-287, 1984. doi: 10.1007/BF01876417.

[13] J. Gutowski, S. Ivanov, and G. Papadopoulos. Deformations of generalized calibrations and compact non-Kähler manifolds with vanishing first Chern class. Asian J. Math., 7(1):39-79, 2003. doi:10.4310/AJM.2003.v7.n1.a4.

[14] R. Harvey and H. B. Lawson, Jr. Calibrated geometries. Acta Math., 148:47-157, 1982. doi:10.1007/BF02392726.

[15] G. R. Jensen and M. Rigoli. Twistor and Gauss lifts of surfaces in four-manifolds. In Recent developments in geometry (Los Angeles, CA, 1987), volume 101 of Contemp. Math., pages 197-232. Amer. Math. Soc., Providence, RI, 1989. doi:10.1090/conm/101/1034983.

[16] K. Kommerell. Riemannsche Flächen im ebenen Raum von vier Dimensionen. Math. Ann., 60(4):548-596, 1905. doi:10.1007/BF01561096.

[17] S. Kwietniewski. Uber Flächen der 4-dimensionalen Raumes deren Tangentialebenen paarweise isoklin sind. $\mathrm{PhD}$ thesis, University of Zürich, 1902.

[18] B. Loo. The space of harmonic maps of $S^{2}$ into $S^{4}$. Trans. Amer. Math. Soc., 313(1):81-102, 1989. doi:10.2307/2001066.

[19] J. D. Lotay. Ruled Lagrangian submanifolds of the 6-sphere. Trans. Amer. Math. Soc., 363(5):2305-2339, 2011. doi:10.1090/S0002-9947-2010-05167-0.

[20] F. Lubbe and L. Schäfer. Pseudo-holomorphic curves in nearly Kähler manifolds. Differential Geom. Appl., 36:24 - 43, 2014. URL: http://www.sciencedirect.com/science/article/pii/ S0926224514001065, doi:https://doi.org/10.1016/j.difgeo.2014.07.002.

[21] N. R. O'Brian and J. H. Rawnsley. Twistor spaces. Ann. Global Anal. Geom., 3(1):29-58, 1985. doi:10.1007/BF00054490.

[22] S. Salamon. Quaternionic Kähler manifolds. Invent. Math., 67(1):143-171, Feb 1982. doi: 10.1007/BF01393378.

[23] S. Salamon. Harmonic and holomorphic maps. In Geometry seminar "Luigi Bianchi" II1984, volume 1164 of Lecture Notes in Math., pages 161-224. Springer, Berlin, 1985. doi: 10.1007/BFb0081912.

[24] R. Storm. Lagrangian submanifolds of the nearly Kähler full flag manifold $F_{1,2}\left(\mathbb{C}^{3}\right)$. arXiv e-prints, page arXiv:1907.06897, Jul 2019. arXiv:1907.06897.

KU Leuven, Department of Mathematics, Celestijnenlaan 200B - Box 2400, Be-3001 Leuven, Belgium

E-mail address: reinier.storm@kuleuven.be 